\title{
Diffusion Tensor Imaging in Preclinical Huntington's Disease
}

\author{
Vincent A. Magnotta • Jinsuh Kim • Tim Koscik • \\ Leigh J. Beglinger • Daisy Espinso • Doug Langbehn • \\ Peg Nopoulos • Jane S. Paulsen
}

Received: 18 March 2008 / Accepted: 6 October 2008/Published online: 21 November 2008

(C) The Author(s) 2008. This article is published with open access at Springerlink.com

\begin{abstract}
Diffusion tensor imaging was used to study brain related changes in white matter that may be associated with Huntington's Disease progression. Thirty-one preclinical gene-mutation carriers were imaged cross-sectionally using diffusion tensor and anatomical brain imaging. Subjects were individuals who had a known gene mutation for HD but did not manifest motor diagnostic criteria for HD. Fractional anisotropy scalar maps showed a positive correlation with five year probability of diagnosis (based upon gene repeat length and current age) in the putamen and a negative correlation in the external capsule. This study shows that scalar maps generated from diffusion tensor imaging may be directly related to the earliest stages of disease progression within HD, even before a diagnosis is given. Findings suggest that DTI measures, therefore,
\end{abstract}

V. A. Magnotta $\cdot$ J. Kim $\cdot$ D. Espinso

Department of Radiology, The University of Iowa,

Iowa City, IA 52245, USA

V. A. Magnotta $\cdot$ L. J. Beglinger $\cdot$ D. Langbehn · P. Nopoulos

J. S. Paulsen

Department of Psychiatry, The University of Iowa,

Iowa City, IA 52245, USA

T. Koscik J. S. Paulsen

Department of Neuroscience, The University of Iowa,

Iowa City, IA 52245, USA

V. A. Magnotta $\cdot$ D. Espinso

Department of Biomedical Engineering, The University of Iowa, Iowa City, IA 52245, USA

\section{J. S. Paulsen $(\bowtie)$}

Departments of Psychiatry, Neurology, and Psychology,

The University of Iowa,

Iowa City, IA 52242-1000, USA

e-mail: jane-paulsen@uiowa.edu may have the ability to act as a biomarker for disease progression in clinical trials of pre-manifest subjects.

Keywords Diffusion tensor imaging .

Fractional anisotropy · Huntington's disease ·

Preclinical · Presymptomatic

\section{Introduction}

Huntington's disease (HD) is an important neurodegenerative disease by virtue of its hereditary nature, grave clinical manifestations and devastating impact on families. HD is an autosomal dominantly inherited disorder characterized by progressive abnormalities of motor and emotional control, cognitive ability, and by involuntary (choreoathetoid) movements. The disease typically strikes in the fourth decade of life, but there is a wide range in age of onset, from childhood to greater than 75 years (Martin 1984). The typical duration from disease diagnosis to death is 15 to 20 years. Neurodegeneration in the HD brain primarily affects the caudate and putamen, but widespread neuronal loss in the cortex and other brain regions have been reported (Cudkowicz \& Kowall 1990). The primary radiographic features of HD are caudate and putamen atrophy which can be measured prior to the motor manifestation of disease in some genemutation carriers (Paulsen et al. 2007). Although more widespread imaging abnormalities had been noted for over a decade (Jernigan et al. 1991) involvement of the extrastriatal measures did not receive much attention until recently. Several publications from different imaging groups have noted disproportionate white matter loss compared to grey matter volume in HD (Aylward et al. 1998; Beglinger et al. 2005; Rosas et al. 2003). Importantly, Beglinger and her colleagues reported that significant white matter abnor- 
mality was more highly associated with cognitive impairment than striatal atrophy, suggesting a particular importance for white matter integrity in the clinical manifestations of HD (Beglinger et al. 2005).

Given the white matter reduction consistently cited across studies, a few groups have recently examined whether a noninvasive modality capable of probing early changes of injured myelin and axons could be beneficial in characterizing early brain changes associated with HD. Diffusion tensor magnetic resonance imaging (DT-MRI) is a recent development providing detailed information about the degree of coherence of neuronal fiber alignment, microstructural integrity, and potentially of white matter connectivity of the brain in vivo. In a diffusion-weighted MR image (DWI), image contrast is determined by the Brownian motion of water molecules (Le Bihan et al. 1986). Anisotropic water diffusion is a property of preferential molecular diffusion in the direction of the fibers running in parallel. In contrast, diffusion is relatively restricted across them. Normal white matter tracts with coherently oriented fibers show high diffusion anisotropy. The basis for changes in diffusion tensor signal is related to the underlying fiber architecture and density, coherence of nerve fiber orientation, and axonal membranes. Measurable decreases in anisotropy have been observed in many pathological processes that cause changes at the microstructural level, such as demyelination and corruption of microtubules, leading to diminished intravoxel fiber coherence. In DT-MRI, diffusion anisotropy effects of DWI data are further exploited utilizing a number of diffusionsensitizing gradients with different angular orientations in order to encode axonal fiber directions (Basser 1995). Diffusion tensor imaging has been applied to studies of brain diseases including cerebral ischemia and infarction, white matter degenerative disorders, Alzheimer's disease (AD), mild cognitive impairment (MCI), multiple sclerosis (MS), traumatic brain injury, and schizophrenia. For example, in MS, diffusion tensor scalars have been shown to reflect axonal attenuation and demyelination (Hesseltine et al. 2006). Work by Parente et al. using DT-MRI to study $\mathrm{AD}$ and MCI (Parente et al. 2008), however, showed that wallerian degeneration secondary to cortical neuronal loss is the primary reason for white matter changes in $\mathrm{AD}$, while myelin breakdown, white matter rarefaction, axonal damage, and gliosis are likely the cause of white matter changes associated with MCI. These studies indicate that DT-MRI can provide insights into pathophysiological mechanisms, even in cases with early disease, such as MCI.

A few recent studies have examined DTI in manifest and preclinical HD. Reading et al. (Reading et al. 2005) first employed the use of DT-MRI to examine changes in white matter in small number of preclinical HD subjects $(n=7)$ as compared to normal controls $(n=7)$. This study employed the use of statistical parametric mapping to evaluate changes in Fractional Anisotropy (FA) and found decreased FA in the superior and middle frontal white matter, pre- and post-central white matter, and a small area in the right occipital lobe. Rosas et al. (2006) and Seppi et al. (Seppi et al. 2006) also employed diffusion tensor imaging to evaluate brain changes associated with HD. Seppi et al. evaluated the trace of the diffusion tensor within subcortical regions of interest and showed an increase in the trace that was related to disease progression in stage I and II manifest HD (Seppi et al. 2006). Rosas et al. (2006) evaluated fractional anisotropy in manifest and preclinical HD subjects as compared to matched control samples. Regions of interest were measured for the corpus callosum, internal capsule, external capsule, and basal ganglia. An exploratory voxel based analysis was also conducted. Fractional anisotropy was reduced within the corpus callosum and the posterior limb of the internal capsule and increases were seen in the anterior limb of the internal capsule, putamen and globus pallidus.

The purpose of this study was to replicate and extend the findings already reported using diffusion tensor derived measures in a larger sample of preclinical HD participants and to assess the strength of the associations between DTI measures and disease progression parameters. We specifically wanted to examine the variation in DTI in the disease epoch prior to diagnosis. Such information would inform efforts to determine markers of disease progression prior to receiving a diagnosis of manifest disease (when functional and staging measures are traditionally used in clinical trials). The estimated five year probability of disease diagnosis was calculated for each preclinical HD subject using a validated formula based upon CAG repeat length and current age (Langbehn et al. 2004).

\section{Methods}

Subjects

The study consisted of 31 subjects who had previously undergone elective independent genetic testing and been found to have the HD gene mutation. Subjects were recruited from the HD Registry at the University of Iowa. Subjects were excluded if they had a history of learning disorder or mental retardation, unstable medical or psychiatric illness, history of alcohol or drug abuse within the previous year, history of other central nervous system (CNS) disease or event, or pacemaker or metallic implants. Diagnosis of HD was determined by a rating of 4 on the motor portion of the Unified Huntington's Disease Rating Scale (UHDRS) following an examination by a movement disorder specialist. Any gene-expanded participant from the 
HD registry who scored a rating of 4 on the UHDRS was excluded from the protocol, therefore all participants were pre-diagnosis or considered preclinical HD. All participants signed a written informed consent approved by the University of Iowa institutional review board before enrollment.

\section{MRI acquisition}

Subjects underwent an imaging protocol that collected high resolution anatomical images and a diffusion tensor sequence. Images were obtained on a $1.5 \mathrm{~T}$ GE Signa $\mathrm{CV} / \mathrm{i} \mathrm{MR}$ scanner. The high resolution anatomical images consisted of a $\mathrm{T} 1$ weighted volume and proton density (PD)/T2 images collected with a dual echo fast spin-echo two dimensional (2D) sequence. The T1 weighted images were acquired in the axial plane using a spoiled grass (SPGR) sequence with the following parameters: echo time $(\mathrm{TE})=3 \mathrm{~ms}$, repetition time $(\mathrm{TR})=24 \mathrm{~ms}$, flip angle $=20^{\circ}$, number of excitations $(\mathrm{NEX})=1$, field of view $(\mathrm{FOV})=$ $240 \times 180 \times 192 \mathrm{~mm}$, matrix $=256 \times 192 \times 124(9 \mathrm{~min}, 30 \mathrm{~s})$. The PD and T2 weighted images were acquired coronally using a fast spin-echo dual-echo sequence with the following parameters: $\mathrm{TE}=36$ (PD) / 96 (T2) $\mathrm{ms}, \mathrm{TR}=$ $3,000 \mathrm{~ms}, \mathrm{NEX}=1, \mathrm{FOV}=260 \times 195 \mathrm{~mm}$, Matrix $=256 \times$ 192, slice thickness/gap $=3.0 / 0.0 \mathrm{~mm}$, and echo train length $=6$ (4 $\mathrm{min}, 30 \mathrm{~s})$. The diffusion tensor data was acquired using an echo-planar spin-echo sequence in the axial plane with the following parameters: $\mathrm{TE}=100 \mathrm{~ms}$, $\mathrm{TR}=6,000 \mathrm{~ms}, \mathrm{FOV}=256 \times 256 \mathrm{~mm}$, Matrix $=128 \times 128$, slice thickness $/$ gap $=4.0 / 0.0 \mathrm{~mm}$, number of diffusion directions $=6$, and B-values $=250,500,750,1,000(2 \mathrm{~min}$, $30 \mathrm{~s})$.

\section{Image analysis}

Processing of the anatomical images was performed using the BRAINS (Brain Research: Analysis of Images, Networks, and Systems) software (Magnotta et al. 2002). The images were processed using a standard image analysis pipeline including anterior commissure (AC)-posterior commissure (PC) alignment, defining of Talairach parameters to warp the Talairach grid onto the subject of interest, tissue classification (Harris et al. 1999), and skull stripping using an artificial neural network (Magnotta et al. 1999). The artificial neural network was also used to define regions of interest for the putamen and caudate in subject space (Powell et al. 2006).

The diffusion tensor data was analyzed using the Guided Tensor Restored Anatomical Connectivity Tractography (GTRACT) (Cheng et al. 2006) software. The diffusion weighted images were co-registered to the B0 image to account for motion and eddy current artifacts. A mutual information metric (D. Mattes et al. 2003; D Mattes et al. 2001) was used to account for differences in signal intensity between the B0 image and the images with larger B values. Next the diffusion tensor was estimated from the diffusion weighted images after applying a $3 \times 3 \times 3$ voxel median filter to the B0 and diffusion weighted images. Diffusion tensor rotational invariant scalar images of fractional anisotropy (FA) and mean diffusivity (MD) were generated. The B0 image was then co-registered with the AC-PC aligned T1 weighted image using a two step registration procedure. First, a six parameter rigid registration (three translations, three rotations) was used to co-register the B0 image with the T1 weighted image. Next, a non-linear B-spline registration was initialized with the rigid transform and used to remove susceptibility artifacts from the EPI images (Kabus et al. 2004). The transformations resulting from the non-linear registration were then used to resample the rotationally invariant scalar measures and the tissue classified image into the same space as the T1 weighted images.

\section{Diffusion tensor scalar analysis}

The AC-PC aligned image for each subject was coregistered with the Montreal Neurological Institute (MNI) 152 atlas image using SPM5. The individual T1 weighted scans were warped into the space of the MNI 152 atlas brain. The resulting deformation field was also used to warp the FA, MD and tissue classified images that were co-registered with the anatomical T1 weighted images. After registration to the MNI brain, the FA, MD and tissue classified images were analyzed using a voxel based analysis. A $2 \mathrm{~mm}$ spatial filter was used to eliminate residual anatomic variability between subjects. Linear regressions were performed to determine the relationship between the image signal intensity and the five year probability of diagnosis within the next five years. This analysis was carried out separately for the FA, MD and tissue classified image sets. The probability of diagnosis is a function of a subject's CAG-expansion length and current age and was calculated using the survival analysis formula of Langbehn et al. (Langbehn et al. 2004). We used this diagnosis probability instead of alternatives such as estimated years to diagnosis because, empirically, diagnosis probability has consistently yielded a more linear relationship with other clinical correlates of HD development using the PREDICT-HD data (Paulsen et al. 2006; Paulsen et al. 2007). PREDICT-HD is a multisite study of over 1,000 participants that investigates the nature and pattern of neurobiological changes and neurobehavioral changes that occur in the period leading up to a diagnosis of Huntington's disease. Manually defined landmark points were placed in the anterior, middle, and posterior portions of the external capsule. This region had 
previously been shown to exhibit lower FA in patients with Huntington's disease as compared to normal controls. A $3 \times 3 \times 3 \mathrm{~mm}$ region was defined using the landmark as the center of the region. FA was measured within these $9 \mathrm{~mm}^{3}$ locations.

\section{Results}

The quality of the registration is shown in Fig. 1. This figure shows nine randomly selected subjects from the study. The spatially normalized and smoothed FA maps are all shown at the same axial slice. The voxel wise analysis showed two main findings for FA. The first was that there was significantly decreased fractional anisotropy in the external capsule bilaterally that was negatively correlated with the probability of diagnosis $(p<0.001$, uncorrected). That is, the FA decreased as the five-year expected probability of diagnosis increased (Fig. 2a). The follow-up ROI analysis using the landmark points within the external capsule did not find any statistically significant differences in the external capsule for any of the regions. The correlations were $-0.19,-0.18$, and -0.17 for the anterior, middle and posterior locations respectively. There was also a significant positive association between the five year expected probability of diagnosis and FA within the putamen bilaterally ( $p<0.001$, uncorrected). That is, that FA increased as the five year probability of diagnosis increased (Fig. 2b). As a follow up analysis to the findings in the putamen, a region of interest (ROI) analysis was conducted using the neural networked defined regions of the putamen derived from the anatomical images. The average FA within the putamen was measured in the space of each subject and correlated with the probability of diagnosis. This resulted in correlations of 0.56 and 0.60 for the right and left putamen (Fig. 3) respectively confirming the voxel based results from this region. Both correlations have a $\mathrm{p}=.001$ by 2 -sided 29 d.f.

Fig. 1 Composite image of nine subjects from the study showing the FA image same axial slice. The axial FA images are shown after smoothing and nonlinear registration to the MNI152 atlas space

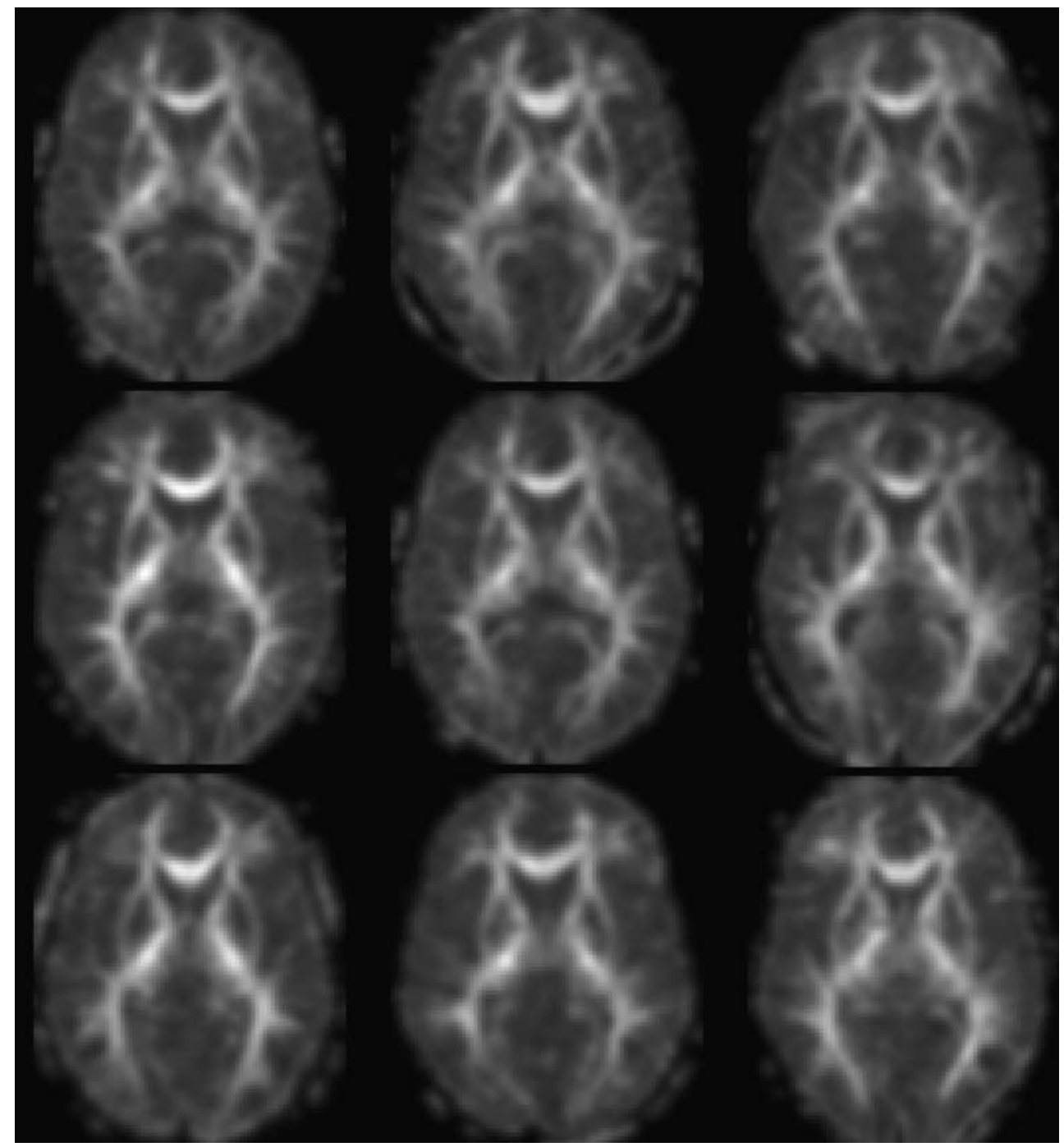


Fig. 2 The top row shows areas where the FA values are positively related to the 5 year probability of onset $(p<0.001$, uncorrected). This is shown overlaid on the a MNI 152 atlas, b mean FA with no smoothing, and $\mathbf{c}$ mean FA with a $2 \mathrm{~mm}$ gaussian filter. The bottom row contains areas where FA was negatively related to the probability of onset ( $p<0.001$, uncorrected). This was mainly confined to the external capsule. The area of significant changes are shown from left to right overlaid on the d MNI 152 atlas, e the mean FA map with no smoothing, and $\mathbf{f}$ the mean FA map with a $2 \mathrm{~mm}$ gaussian filter

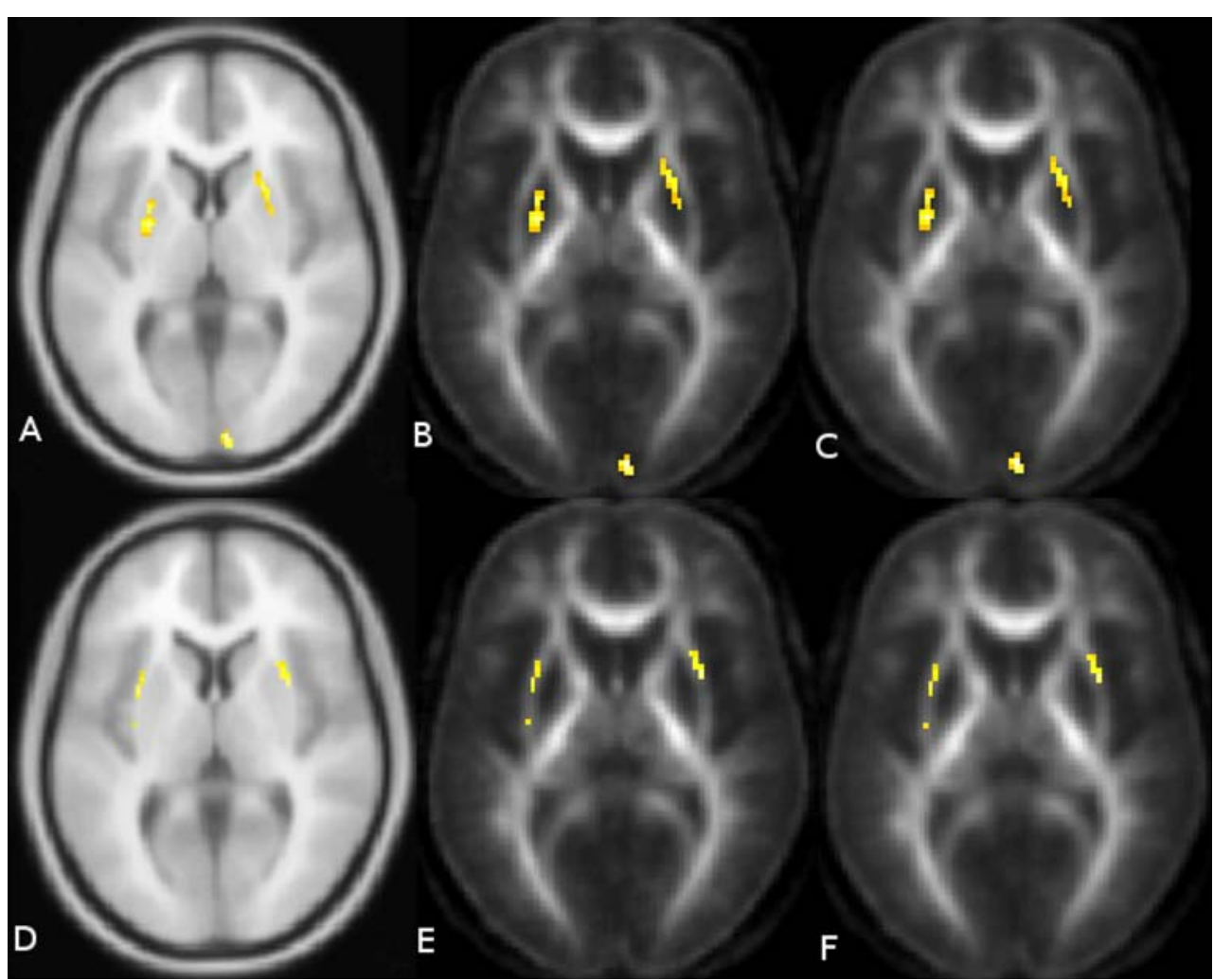

t-test against the null hypothesis of no association. Two rather extreme observations existed in the data. Therefore, the robustness of the linear correlations was verified by conservatively recalculating after removing these two points. Although the magnitude and statistical significance of the correlations are somewhat reduced, both remain statistically significant at levels between .01 and .02. The results of the mean diffusivity also showed a positive correlation with probability of diagnosis in both the caudate and putamen bilaterally (Fig. 4). This means that water mobility within these subcortical structures increases as one progresses towards disease diagnosis. There were no negative correlations between MD and probability of diagnosis. Finally, the tissue classification shows changes in the border between the caudate and ventricles and the border between the putamen and external capsule (Fig. 5). This is consistent with other widely-reported evidence that the size of the caudate and putamen are shrinking as the subjects progress towards clinical manifestation of HD. Follow-up region of interest a

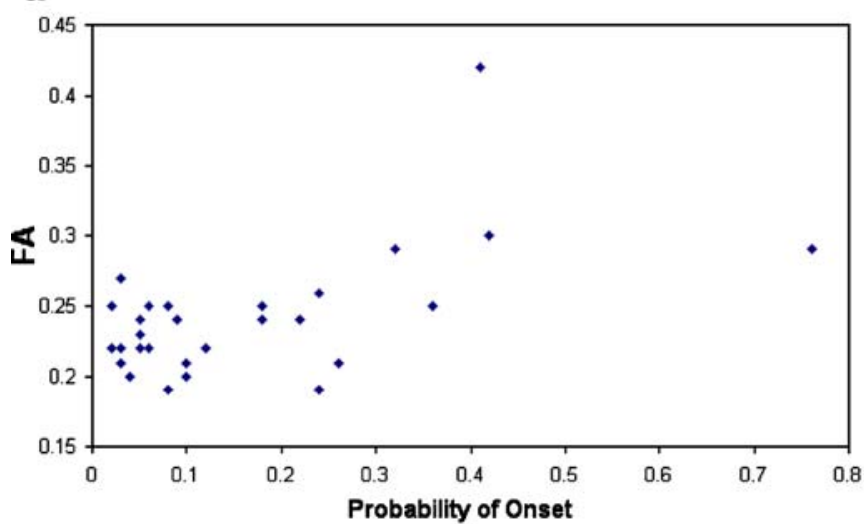

b

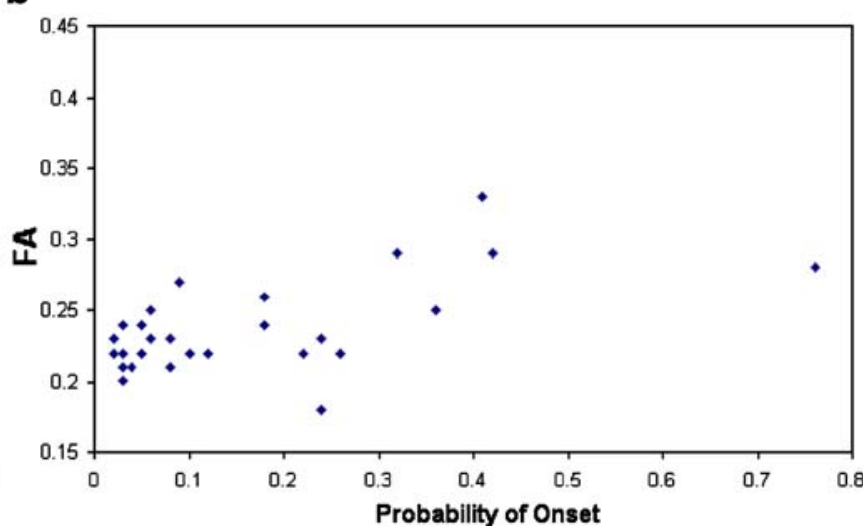

Fig. 3 Relationship between the probability of onset and the average FA values within the caudate and putamen using a region of interest approach. Scatter plots are shown for the left (a) and right (b) putamen 
Fig. 4 Shows the regions with increased mean diffusivity $(p<$ 0.001 uncorrected). Regions meeting this criteria include the a cerebellum, b striatum, and $\mathbf{c}$ cortical regions

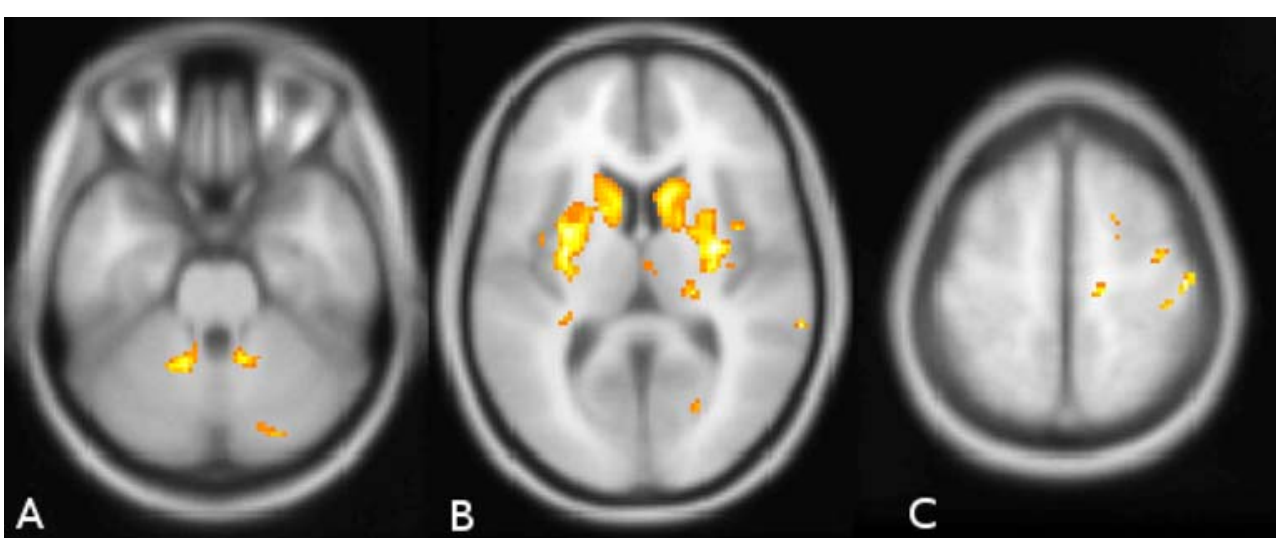

analysis of the size and mean diffusivity within the caudate and putamen confirmed this finding. The caudate volume showed the greatest correlation with probability of diagnosis $(-0.77)$ while the putamen correlation was -0.40 . The mean diffusivity within the caudate and putamen were very similar and had positive correlations between 0.64 and 0.69 for both regions of interest. All positive and negative relationships are shown by region of interest in the tables.

\section{Discussion}

The use of diffusion tensor imaging in preclinical HD supports and extends the findings of previous brain morphology studies showing changes in white matter before the diagnosis of manifest motor disease. A number of previous studies have shown reduced white matter volume and abnormal DTI measures in preclinical subjects with the Huntington gene expansion (Aylward et al. 1998; Beglinger et al. 2005; Nopoulos et al. 2007;

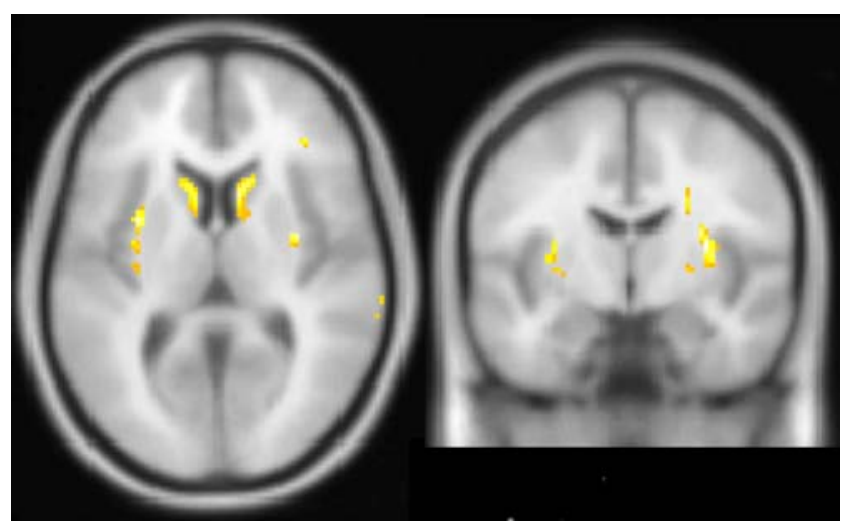

Fig. 5 Regions of tissue type changes associated with disease progression $(p<0.001$, uncorrected). These changes are mainly confined to the border between the caudate/ventricle and between the putamen/external capsule
Rosas et al. 2003). Reading et al. found focal changes within the superior frontal, middle frontal and postcentral white matter (Reading et al. 2005). The work by Rosas et al. (2006) compared preclinical HD subjects with normal controls and found decreased FA within the corpus callosum and internal capsule as well as increased FA in the putamen and globus pallidus. A voxel based analysis of these same subjects showed a similar pattern of changes and included decreased FA in the external capsule and thalamus. Mean diffusivity was also shown to increase in the putamen and globus pallidus in the subjects with clinically diagnosed HD as well as the preclinical HD subjects as compared to control subjects. No MD changes were found in the caudate in a region of interest analysis. While the current study replicates and extends previous DTI abnormalities by using a larger sample size (over twice the $n$ of previous studies), we also confirm a significant relationship between these diffusion tensor scalar measures and estimated proximity to HD diagnosis. The confirmation of this finding is key to current efforts to establish possible markers of disease progression for use in preclinical experimental trials. This study does differ from the work of Rosas et al. in that a positive relationship between mean diffusivity and probability of diagnosis was found in the caudate as well as the putamen, while no changes related to disease progression were manifested in the globus pallidus.

While this study shows an interesting relationship between the probability of diagnosis and diffusion tensor scalar measures, one limitation of the study is the use of voxel based morphometry as the primary analysis tool. In this approach, one is measuring residual changes in the image modalities after nonlinear registration. Diseases that change brain morphometry may therefore be susceptible to identifying regions that appear different simply as a result of increased anatomical variability that is not compensated by the image registration procedure. In this study, changes in the FA within the external capsule are susceptible to this artifact and we are unable to replicate the VBM results in 
Table 1 Locations with a significant positive relationship ( $p<0.001$, uncorrected) between FA and five year probability of onset

\begin{tabular}{lrrr}
\hline Region & \multicolumn{1}{c}{ X } & \multicolumn{1}{c}{ Y } & Z \\
\hline Right Lingual Gryus & 8 & -96 & 2 \\
Left Putamen & -26 & 2 & 4 \\
Right Putamen & 24 & 14 & 2 \\
Right Insula & 38 & -18 & 8 \\
Left Middle Frontal Gyrus & -8 & 58 & 18 \\
Left Middle Frontal Gyrus & -42 & 48 & 16 \\
Left Potserior Cingulate & -20 & -62 & 12 \\
Left Medial Frontal Gyrus & -6 & -10 & 60 \\
\hline
\end{tabular}

the external capsule in a follow-up ROI analysis. Therefore, the white matter changes within this region appear to be the result of residual anatomical variability. The VBM based finding of changes in fractional anisotropy and mean diffusivity within the caudate and putamen, however, were followed up with an ROI analysis that confirmed the findings. We cannot rule out, however, that slight misalignment of the B0 images with the anatomical data or image distortion of the echo-planar image could result in increased FA within the putamen by placement of the external capsule within the regions of interest defined on the anatomical images.

Decreased volume of the striatum has been documented to occur in preclinical HD individuals many years out from diagnosis (Aylward et al. 2004). While the increased FA (interpreted as higher white matter integrity) in the putamen may seem counterintuitive, a reasonable explanation is that the loss of grey matter in this region produces a greater white matter / grey matter ratio. Increased mean diffusivity has been shown to be related to demyelination axonal loss, and gliosis. A classic Huntington's disease manifestation includes both gliosis and neural loss within the basal ganglia region. This clearly needs further study. Recent work by Bartzokis et al. have shown an increase in oligodendrocytes years in advance of the clinical manifestation of the illness (Bartzokis et al. 2007). The oligoden-

Table 2 Locations with a significant negative relationship $(p<0.001$, uncorrected) between FA and five year probability of onset

\begin{tabular}{lrrr}
\hline Region & \multicolumn{1}{c}{ X } & Y & Z \\
\hline Right Middle Frontal Gyrus & 18 & 6 & 50 \\
Right External Capsule & 32 & 8 & 4 \\
Left External Capsule & -30 & 6 & 10 \\
Left External Capsule & -32 & -10 & 12 \\
Right Anterior Frontal WM & 18 & 20 & 40 \\
\hline
\end{tabular}

Table 3 Locations with a significant positive relationship $(p<0.001$, uncorrected) between mean diffusivity and the five year probability of onset

\begin{tabular}{lrrr}
\hline Region & X & Y & Z \\
\hline Right Putamen & 30 & -6 & 10 \\
Left Putamen & -32 & -10 & 14 \\
Right Post Central Gyrus & 48 & -22 & 52 \\
Left inferior frontal Gyrus & -52 & 24 & 22 \\
Left Cerebellum WM & -12 & -50 & -34 \\
Right Parahippocampal gyrus & 22 & -16 & -14 \\
Right Parietal WM & 22 & -32 & 30 \\
Right Middle Frontal gyrus & 14 & -22 & 62 \\
Right Cerebellum & 12 & -50 & -28 \\
Right Cerebellum & 52 & -58 & -20 \\
\hline
\end{tabular}

drocytes are responsible for axonal myelination repair. However, an increased concentration of these cells may result in free-radical-induced tissue damage due to the large intracellular iron content. This may also explain the increased FA within the putamen. It has been shown that the transition zone between the putamen and globus pallidus has a large concentration of oligodendrocytes in primates (Cammermeyer 1960).

\section{Conclusion}

This study shows that scalar maps generated from diffusion tensor imaging may be directly related to the earliest stages of disease progression within HD, even before a diagnosis is warranted. Findings suggest that DTI measures, therefore, may have the ability to act as a biomarker for disease progression in clinical trials of premanifest subjects.

Tables 1, 2, 3, and 4 .

Table 4 Locations with significant changes $(p<0.001$, uncorrected) in tissue classification related to five year probability of onset

\begin{tabular}{lrrr}
\hline Region & X & Y & Z \\
\hline Left External Capsule & -32 & 4 & 10 \\
Right WM around Putamen & 30 & -8 & 16 \\
Right Parietal Lobe WM & 36 & -64 & 18 \\
Right Caudate & 12 & 12 & 12 \\
Left Middle Frontal Gyrus & -52 & 24 & 22 \\
Right Post Central Gyrus & 50 & -20 & 56 \\
Left Frontal WM & -26 & 12 & 28 \\
Left Caudate & -10 & 6 & 0 \\
Right External Capsule & 24 & -4 & 32 \\
Right External Capsule & 28 & 18 & 22 \\
Right Frontal Lobe WM & 36 & 34 & 10 \\
\hline
\end{tabular}


Acknowledgements Supported by funding from grants NS40068 and NS050568 from the National Institute on Neurological Disorders and Stroke, the Roy J. Carver Charitable Trust, the Howard Hughes Medical Institute, and the High Q Foundation.

Open Access This article is distributed under the terms of the Creative Commons Attribution Noncommercial License which permits any noncommercial use, distribution, and reproduction in any medium, provided the original author(s) and source are credited.

\section{References}

Aylward, E. H., Anderson, N. B., Bylsma, F. W., Wagster, M. V., Barta, P. E., Sherr, M., et al. (1998). Frontal lobe volume in patients with Huntington's disease. Neurology, 50(1), 252-258.

Aylward, E. H., Sparks, B. F., Field, K. M., Yallapragada, V., Shpritz, B. D., Rosenblatt, A., et al. (2004). Onset and rate of striatal atrophy in preclinical Huntington disease. Neurology, 63(1), 66-72.

Bartzokis, G., Lu, P. H., Tishler, T. A., Fong, S. M., Oluwadara, B., Finn, J. P., et al. (2007). Myelin breakdown and iron changes in Huntington's disease: pathogenesis and treatment implications. Neurochemical Research, 32(10), 1655-1664. doi:10.1007/ s11064-007-9352-7.

Basser, P. J. (1995). Inferring microstructural features and the physiological state of tissues from diffusion-weighted images. NMR in Biomedicine, 8, 333-344. doi:10.1002/nbm.1940080707.

Beglinger, L., Nopoulos, P., Jorge, R., Langbehn, D., Mikos, A., Moser, D., et al. (2005). White matter volume and cognitive dysfunction in early Huntington's disease. Cognitive and Behavioral Neurology, 18(2), 102-107. doi:10.1097/01.wnn.0000152205.79033.73.

Cammermeyer, J. (1960). The distribution of oligodendrocytes in cerebral gray and white matter of several mammals. The American Journal of Anatomy, 107, 107-127. doi:10.1002/aja.1001070203.

Cheng, P., Magnotta, V. A., Wu, D., Nopoulos, P., Moser, D. J., Paulsen, J., et al. (2006). Evaluation of the GTRACT diffusion tensor tractography algorithm: A validation and reliability study. NeuroImage, 31(3), 1075-1085. doi:10.1016/j.neuroimage.2006.01.028.

Cudkowicz, M., \& Kowall, N. W. (1990). Degeneration of pyramidal projection neurons in Huntington's disease cortex. Annals of Neurology, 27, 200-204. doi:10.1002/ana.410270217.

Harris, G., Andreasen, N. C., Cizadlo, T., Bailey, J. M., Bockholt, H. J., Magnotta, V. A., et al. (1999). Improving tissue classification in MRI: a three-dimensional multispectral discriminant analysis method with automated training class selection. Journal of Computer Assisted Tomography, 23(1), 144-154. doi:10.1097/ 00004728-199901000-00030.

Hesseltine, S. M., Law, M., Babb, J., Rad, M., Lopez, S., Ge, Y., et al. (2006). Diffusion tensor imaging in multiple sclerosis: assessment of regional differences in the axial plane within normalappearing cervical spinal cord. AJNR. American Journal of Neuroradiology, 27(6), 1189-1193.

Jernigan, T. L., Salmon, D. P., Butters, N., \& Hesselink, J. R. (1991). Cerebral structure on MRI, Part II: Specific changes in Alzheimer's and Huntington's diseases. Biological Psychiatry, 29(1), 68-81. doi:10.1016/0006-3223(91)90211-4.

Kabus, S., Netsch, T., Fischer, B., \& Modersitzki, J. (2004). B-Spline registration of 3D images with Levenberg-Marquardt optimization. Proc. of SPIE Medical Imaging, 5370, 304-313.

Langbehn, D., Brinkman, R., Falush, D., Paulsen, J., \& Hayden, M. (2004). A new model for prediction of the age of onset and penetrance for Huntington's disease based on CAG length. Clinical Genetics, 65, 267-277. doi:10.1111/j.1399-0004.2004.00241.x.

Le Bihan, D., Breton, E., Lallemand, D., Grenier, P., Cabanis, E., \& Laval-Jeantet, M. (1986). MR imaging of intravoxel incoherent motions: Application to diffusion and perfusion in neurologic disorders. Radiology, 161(2), 401-407.

Magnotta, V. A., Harris, G., Andreasen, N. C., O’Leary, D. S., Yuh, W. T., \& Heckel, D. (2002). Structural MR image processing using the BRAINS2 toolbox. Computerized Medical Imaging and Graphics, 26(4), 251-264. doi:10.1016/S0895-6111(02) 00011-3.

Magnotta, V. A., Heckel, D., Andreasen, N. C., Cizadlo, T., Corson, P. W., Ehrhardt, J. C., et al. (1999). Measurement of brain structures with artificial neural networks: two- and three-dimensional applications. Radiology, 211(3), 781-790.

Martin, J. B. (1984). Huntington's disease: New approaches to an old problem. Neurology, 34(8), 1059-1072.

Mattes, D., Haynor, D. R., Vesselle, H., Lewellen, T., \& Eubank, W. (2003). PET-CT Image Registration in the Chest Using Free-form Deformations. IEEE Transactions on Image Processing, 22(1), $120-128$.

Mattes, D., Haynor, D. R., Vesselle, H., Lewellen, T. K., \& Eubank, W. (2001). Nonrigid multimodality image registration. In M. Sonka, K. M. Hanson (Eds.), Proc. SPIE vol. 4322, Medical Imaging 2001: Image Processing (pp. 1609-1620).

Nopoulos, P., Magnotta, V., Mikos, A., Paulson, H., Andreasen, N., \& Paulsen, J. (2007). Morphology of the cerebral cortex in preclinical Huntington's disease. The American Journal of Psychiatry, 164(9), 1428-1434. doi:10.1176/appi.ajp.2007.06081266.

Parente, D. B., Gasparetto, E. L., da Cruz Jr, L. C., Domingues, R. C., Baptista, A. C., Carvalho, A. C., et al. (2008). Potential role of diffusion tensor MRI in the differential diagnosis of mild cognitive impairment and Alzheimer's disease. AJR. American Journal of Roentgenology, 190(5), 1369-1374. doi:10.2214/AJR.07.2617.

Paulsen, J. S., Hayden, M., Stout, J. C., Langbehn, D. R., Aylward, E., Ross, C. A., et al. (2006). Preparing for preventive clinical trials: the Predict-HD study. Archives of Neurology, 63(6), 883-890. doi:10.1001/archneur.63.6.883.

Paulsen, J. S., Langbehn, D. R., Stout, J. C., Aylward, E., Ross, C. A., Nance, M., et al. (2007). Detection of Huntington's disease decades before diagnosis: the Predict HD study. J Neurol Neurosurg Psychiatry.

Powell, S., Magnotta, V., Johnson, H., \& Andreasen, N. C.(2006). Automated brain segmentation using neural networks. Paper presented at the SPIE Medical Imaging, San Diego.

Reading, S., Yassa, M., Bakker, A., Dziorny, A., Gourley, L., Yallapragada, V., et al. (2005). Regional white matter change in pre-symptomatic Huntington's disease: a diffusion tensor imaging study. Psychiatry Research, 140(1), 55-62. doi:10.1016/j. pscychresns.2005.05.011.

Rosas, H., Koroshetz, W., Chen, Y., Skeuse, C., Vangel, M., Cudkowicz, M., et al. (2003). Evidence for more widespread cerebral pathology in early HD: an MRI-based morphometric analysis. Neurology, 60(10), 1615-1620.

Rosas, H., Tuch, D., Hevelone, N., Zaleta, A., Vangel, M., Hersch, S., et al. (2006). Diffusion tensor imaging in presymptomatic and early Huntington's disease: Selective white matter pathology and its relationship to clinical measures. Movement Disorders, 21(9), 1317-1325. doi:10.1002/mds.20979.

Seppi, K., Schocke, M., Mair, K., Esterhammer, R., WeirichSchwaiger, H., Utermann, B., et al. (2006). Diffusion-weighted imaging in Huntington's disease. Movement Disorders, 21(7), 1043-1047. doi:10.1002/mds.20868. 